\title{
Fully Integrated Light-Sensing Stimulator Design for Subretinal Implants
}

\author{
Hosung Kang ${ }^{1}$, Wajahat H. Abbasi ${ }^{2}$, Seong-Woo Kim ${ }^{3, *}$ and Jungsuk Kim ${ }^{2,4, *}$ \\ 1 Department of Medical Science, Korea University, Seoul 02841, Korea; 2017010569@korea.ac.kr \\ 2 Department of Health Science and Technology, GAIHST, Incheon 21999, Korea; Waj@bme.gachon.ac.kr \\ 3 Department of Ophthalmology, Korea University Guro Hospital, Korea University College of Medicine, \\ Seoul 08308, Korea \\ 4 Department of Biomedical Engineering, Gachon University, Incheon 21936, Korea \\ * Correspondence: ksw64723@korea.ac.kr (S.-W.K.); jungsuk@bme.gachon.ac.kr (J.K); \\ Tel.: +82-32-820-4430 (J.K)
}

Received: 20 November 2018; Accepted: 24 January 2019; Published: 28 January 2019

check for updates

\begin{abstract}
This paper presents a fully integrated photodiode-based low-power and low-mismatch stimulator for a subretinal prosthesis. It is known that a subretinal prosthesis achieves 1600-pixel stimulators on a limited single-chip area that is implanted beneath the bipolar cell layer. However, the high-density pixels cause high power dissipation during stimulation and high fabrication costs because of special process technologies such as the complementary metal-oxide semiconductor CMOS image sensor process. In addition, the many residual charges arising from the high-density pixel stimulation have deleterious effects, such as tissue damage and electrode corrosion, on the retina tissue. In this work, we adopted a switched-capacitor current mirror technique for the single-pixel stimulator (SPStim) that enables low power consumption and low mismatch in the subretinal device. The customized $\mathrm{P}+/ \mathrm{N}$-well photodiode used to sense the incident light in the SPStim also reduces the fabrication cost. The 64-pixel stimulators are fabricated in a standard $0.35-\mu \mathrm{m}$ CMOS process along with a global digital controller, which occupies a chip area of $4.3 \times 3.2 \mathrm{~mm}^{2}$ and are ex-vivo demonstrated using a dissected pig eyeball. According to measured results, the SPStim accomplishes a maximum biphasic pulse amplitude of $143 \mu \mathrm{A}$, which dissipates an average power of $167 \mu \mathrm{W}$ in a stimulation period of $5 \mathrm{~ms}$, and an average mismatch of $1.12 \%$ between the cathodic and anodic pulses.
\end{abstract}

Keywords: subretinal prosthesis; photodiode; high-density pixels; ex-vivo demonstration; light sensor; digital controller; implantable device

\section{Introduction}

Retinal implants offer great promise for restoring vision to patients who suffer from retinal diseases such as retina pigmentosa and age-related macular degeneration. According to the anatomical position, retinal prostheses can be classified as epiretinal [1,2], subretinal [3-5], or suprachoroidal [6,7] devices. Among them, it has been reported that the subretinal implant can provide high pixel density of up to 1600 pixels [8], on a limited silicon chip area [9]; this is possible because the stimulator in the subretinal device does not need a high-resolution current-steering digital-to-analog converter (DAC) and its own local digital controller, which are employed to generate various biphasic current pulses for epiretinal and suprachoroidal prosthetics. The high-resolution DAC and the digital controller occupy a large area in a single-pixel stimulator (SPStim); the area becomes dominant with high-voltage CMOS (complementary metal-oxide semiconductor) process technology [2].

Figure 1a shows a subretinal prosthesis that is inserted into the subretinal space of the eye via ab-externo approach. The prosthetic chip and cable, which works to deliver a power and a command 
data from an inductive coupling coil located in the retroauricular area, enters the subretinal space passing through the partial-thickness scleral flap. Then, the rest cable outside the eye is buried under the temporalis muscle. Figure $1 \mathrm{~b}$ depicts the general architecture of a single-pixel stimulator (SPStim) for a subretinal implant, which is composed of a photosensor, a current amplifier, and a pulse shaper. A photodiode in the photosensor produces a dark current corresponding to the light intensity incoming onto the retina. The dark current is in the order of nano-amperes and has a monophasic shape. The current amplifier in Figure 1b works to amplify the dark current from tens to hundreds of microamperes. Finally, the pulse shaper reshapes the amplified monophasic signal to a biphasic current pulse, which consists of a rectangular cathodic and anodic pulse, and then delivers the biphasic signal into the bipolar cells interfaced with a microelectrode.

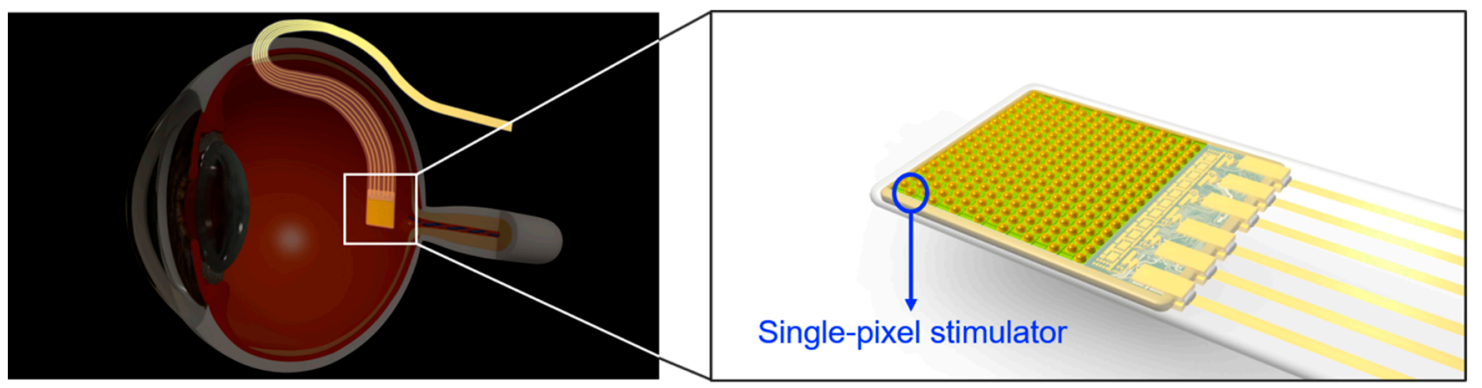

(a)

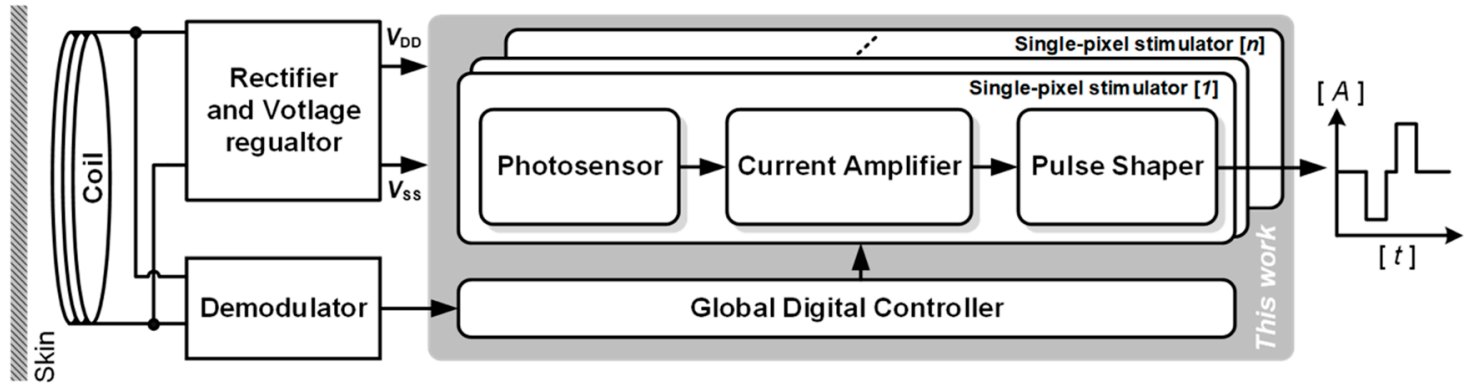

(b)

Figure 1. (a) System overview of a subretinal prosthesis that is implanted beneath the bipolar cell layer, (b) simplified architecture of the subretinal device including the single-pixel stimulator.

The single-pixel stimulator for the subretinal prosthesis should meet three design requirements as follows. First, the amplitude mismatch of the cathodic and anodic pulses should be as low as possible in order to reduce the residual charge on the retina tissue after stimulation. The residual charge sometimes induces unwanted spike excitation that results in tissue damage, as well as electrode corrosion that impedes the delivery of stimulation charge to the tissue from the stimulator output [10]. Thus, it is indispensable to minimize the amplitude mismatch of the biphasic stimulus current. Second, it must be fully controllable by digital logic embedded on the same chip in order to generate the diverse shapes of the biphasic stimulus current. Because visually impaired patients have individually different thresholds for exciting the nerves in the retina [11], after implantation, the stimulus current shapes must be adjusted, including the amplitude, width, period of the biphasic stimuli, and the interphase delay between the cathodic and anodic pulses. Finally, the stimulator necessitates a wide dynamic output range to produce various amplitudes of the biphasic current owing to the different thresholds of the patients to excite their retinal nerves. A simple way to widen the output dynamics is to raise the power supply rail. However, the high-voltage operation leads to high power consumption in the SPStim, which would become worse in a subretinal device with high-density pixels. We thus must devise a new SPStim architecture to accomplish the wide dynamic output range while maintaining low-voltage operation. 
Over the last decade, a few single-pixel stimulators have been presented for subretinal implants to implement high-density stimulator arrays [3], low-voltage operation [12], and low mismatch [6,13]. To the authors' best knowledge, however, any results that meet all of these design requirements have not yet been presented. Thus motivated, in this work we propose a SPStim design that adopts a switched-capacitor current mirror technique that achieves both low mismatch and wide dynamic output range in low-voltage operation. This stimulator circuit was fabricated in a standard $0.35-\mu \mathrm{m}$ 4M2P CMOS process and ex-vivo demonstrated employing a dissected pig eyeball.

\section{Methods}

Figure 2 shows the circuit diagram of the proposed SPStim, which is composed of three stages: (1) a photosensor, (2) a current amplifier, and (3) a pulse shaper, as illustrated in Figure 1b. Also shown are simulated transient waveforms for the SPStim's digital inputs and analog output. The photosensor stage is composed of a photodiode $\mathrm{D}_{\mathrm{PD}}$ and a PMOS transistor $\mathrm{M}_{1}$, which works as a switch to reset $\mathrm{D}_{\mathrm{PD}}$. In this design, a customized photodiode that has the structure of a $\mathrm{P}+$ and $\mathrm{N}$-well as illustrated in Figure 2a has been employed because of its high sensitivity [14] and relatively low fabrication cost compared with the CMOS image sensor process that requires a deeper epitaxial layer, anti-reflective coating and optimization of passivation in order to minimize interference [15]. $\mathrm{D}_{\mathrm{PD}}$ can be modeled as a dark current source $\mathrm{I}_{\mathrm{DARK}}$, which is proportional to the incident light intensity, and a parasitic capacitor $\mathrm{C}_{\mathrm{PD}}$, which arises from the junction area of the $\mathrm{P}+$ and $\mathrm{N}$-well in the diode, $\mathrm{C}_{\mathrm{gd} 1}, \mathrm{C}_{\mathrm{gs} 3}$, $\mathrm{C}_{\mathrm{gs} 2}$, and $\mathrm{C}_{\mathrm{gs} 2}$. Here, $\mathrm{C}_{\mathrm{gd}}$, and $\mathrm{C}_{\mathrm{gs}}$ denote a gate-drain and gate-source parasitic capacitance of the transistors. Although $\mathrm{I}_{\mathrm{DARK}}$ and $\mathrm{C}_{\mathrm{PD}}$ respectively vary with the light intensity and the dimension of $\mathrm{D}_{\mathrm{PD}}$ and its adjacent transistors, we set $\mathrm{I}_{\mathrm{DARK}}$ and $\mathrm{C}_{\mathrm{PD}}$ to $8 \mathrm{nA}$ and $6 \mathrm{pF}$ for the simulation environment.

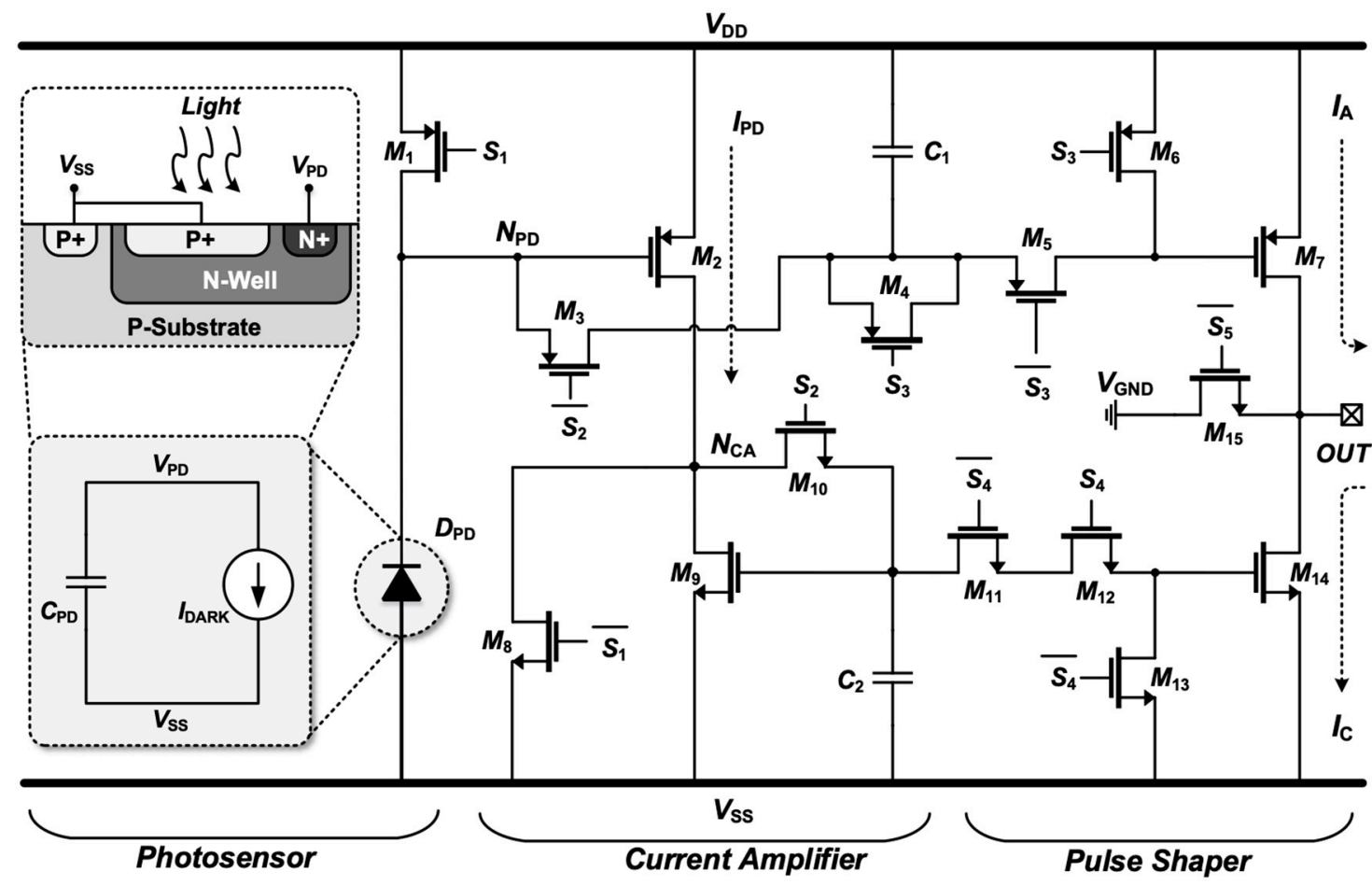

(a)

Figure 2. Cont. 

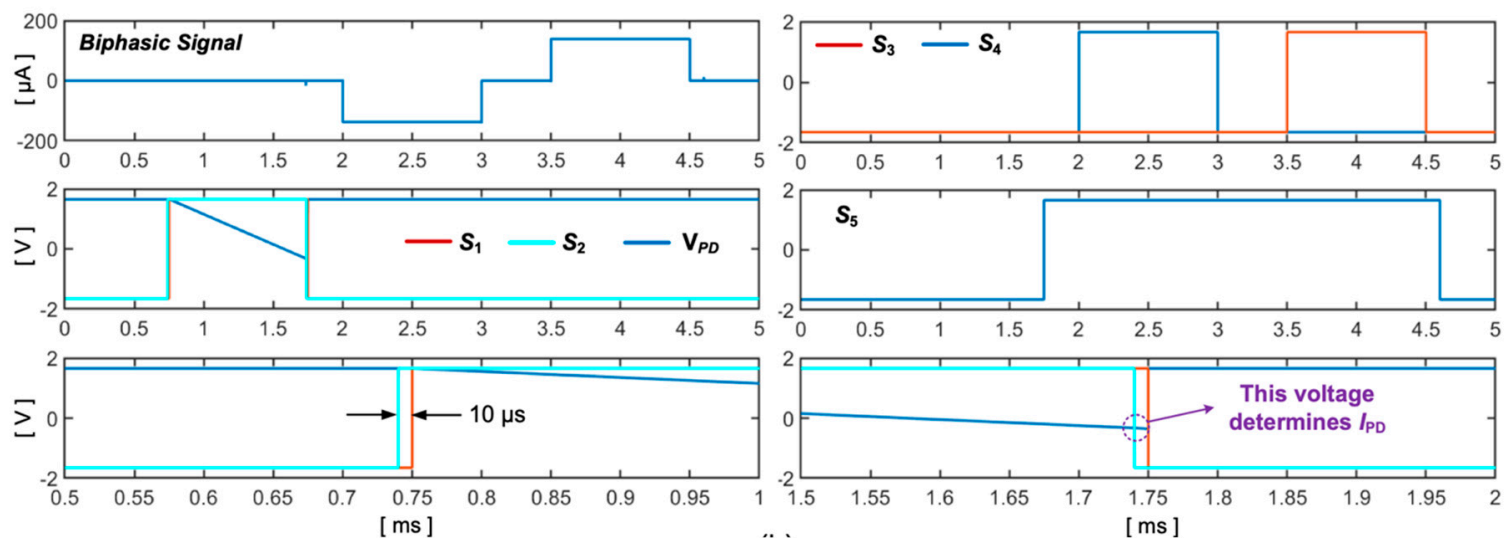

(b)

Figure 2. (a) Circuit diagram of the proposed single-pixel stimulator adopting a customized photodiode and a switched capacitor current mirror technique; (b) simulated transient waveform to show input and analog output.

The proposed SPStim operates in four different modes: the preset mode $(0-0.74 \mathrm{~ms}$ in Figure $2 \mathrm{~b})$, reset mode $(0.74-0.75 \mathrm{~ms})$, charging mode $(0.75-1.75 \mathrm{~ms})$, and stimulation mode $(0.175-4.6 \mathrm{~ms})$, followed by the next preset mode $(0.46-5 \mathrm{~ms})$. During the preset mode, where all signals of the digital controller are set to logic 0 , the PMOS transistor $\mathrm{M}_{1}$ is fully turned on, and as a result, $\mathrm{C}_{\mathrm{PD}}$ is charged up to $\mathrm{V}_{\mathrm{DD}}$. The stages other than the photodiode stage are deactivated in this mode to diminish unnecessary power dissipation. By applying a logic 1 to the $S_{2}$ on $M_{3}$ and $M_{10}$ in the current amplifier stage, the reset mode begins, where the capacitors $C_{1}$ and $C_{2}$ are reset to $V_{D D}$ through $M_{1}$ and $M_{3}$, and to $V_{S S}$ through $M_{8}$ and $M_{10}$, respectively. Thus, the previous stimulation memory recorded on $C_{1}$ and $\mathrm{C}_{2}$ is erased so as to receive new stimulation information.

The charging mode starts when the $S_{1}$ on $M_{1}$ and $M_{8}$ becomes logic 1 . The $S_{1}$ is synchronized with the $S_{2}$, but its rising and falling edges lag those of the $S_{2}$ by $10 \mu \mathrm{s}$. We generate the $S_{1}$ from the $S_{2}$ using a simple delay chain as presented in [16]. As the $S_{1}$ turns high, the transistor $M_{1}$ is immediately disabled. Because of the $\mathrm{I}_{\mathrm{DARK}}$, the node voltage of $\mathrm{N}_{\mathrm{PD}}$, here called $\mathrm{V}_{\mathrm{PD}}$, begins to steadily drop from $\mathrm{V}_{\mathrm{DD}}$ to $\mathrm{V}_{\mathrm{DD}}-\mathrm{I}_{\mathrm{DARK}} \times \Delta \mathrm{T} /\left(\mathrm{C}_{1}+\mathrm{C}_{\mathrm{PD}}\right)$, where $\Delta \mathrm{T}$ indicates the duration of the charging mode (0.75-1.75 ms in this simulation). The drop in $\mathrm{V}_{\mathrm{PD}}$ enables the PMOS transistor $\mathrm{M}_{2}$, thereby entering it to a saturation region. Accordingly, the current $\mathrm{I}_{\mathrm{PD}}$ generated from $\mathrm{M}_{2}$ can be defined as

$$
I_{\mathrm{PD}}=\frac{1}{2} \mu_{\mathrm{P}} C_{\mathrm{OX}}\left(\frac{W}{L}\right)_{2}\left(V_{\mathrm{DD}}-V_{\mathrm{PD}}-\left|V_{\mathrm{TH}, \mathrm{P}}\right|\right)^{2}=\frac{1}{2} \mu_{\mathrm{P}} C_{\mathrm{Ox}}\left(\frac{W}{L}\right)_{2}\left(\frac{I_{\mathrm{DARK}} \times \Delta T}{C_{1}+C_{\mathrm{PD}}}-\left|V_{\mathrm{TH}, \mathrm{P}}\right|\right)^{2}
$$

Here, $\mu_{\mathrm{P}}, C_{\mathrm{OX}}, W / L$, and $V_{\mathrm{TH}, \mathrm{P}}$ are the channel mobility, gate oxide capacitance per unit area, aspect ratio, and PMOS threshold voltage, respectively. This equation shows that the small current $I_{\text {DARK }}$ in the nano-ampere range is amplified to a stimulus current in the tens to hundreds of micro-amperes by adjusting the charging duration $\Delta T$. In this work, we aimed to generate a stimulation current of $150 \mu \mathrm{A}$ at maximum. Because $I_{\mathrm{PD}}$ produced from $\mathrm{M}_{2}$ flows out to $V_{\mathrm{SS}}$ only through $\mathrm{M}_{9}, I_{\mathrm{PD}}$ can be transformed to:

$$
I_{\mathrm{PD}}=\frac{1}{2} \mu_{\mathrm{P}} C_{\mathrm{Ox}}\left(\frac{W}{L}\right)_{2}\left(V_{\mathrm{DD}}-V_{\mathrm{PD}}-\left|V_{\mathrm{TH}, \mathrm{P}}\right|\right)^{2}=\frac{1}{2} \mu_{\mathrm{N}} C_{\mathrm{Ox}}\left(\frac{W}{L}\right)_{9}\left(V_{\mathrm{CA}}-V_{\mathrm{TH}, \mathrm{N}}\right)^{2}
$$


Here, $\mu_{\mathrm{N}}$ and $V_{\mathrm{CA}}$ denote the channel mobility of NMOS transistor and the node voltage of $N_{\mathrm{CA}}$, respectively. $V_{\mathrm{CA}}$ can be rewritten as:

$$
V_{\mathrm{CA}}=\sqrt{\frac{2 I_{\mathrm{PD}}}{\mu_{\mathrm{N}} C_{\mathrm{OX}}\left(\frac{W}{L}\right)_{9}}}+V_{\mathrm{TH}, \mathrm{N}}
$$

Including $I_{\mathrm{PD}}, V_{\mathrm{CA}}$ changes with the incident light intensity and $\Delta T$. Consequently, the voltages $V_{\mathrm{PD}}$ and $V_{\mathrm{CA}}$ are correlated with $I_{\mathrm{PD}}$, which is used as the stimulus current, and are recorded on $C_{1}$ and $C_{2}$ at $1.74 \mathrm{~ms}$ in Figure $2 \mathrm{~b}$ when the $S_{2}$ turns back to a logic 0 . After $10 \mu \mathrm{s}$, the $S_{1}$ turns off again. Here, the 10- $\mu$ s delay reduces the deleterious effect of charge injection that can lead to voltage fluctuations on $C_{1}$ and $C_{2}$ by the $S_{1}$ switching.

The stimulation mode starts when the $S_{5}$ signal turns to a logic 1 at $1.75 \mathrm{~ms}$ in Figure $2 \mathrm{~b}$. In other modes except for this stimulation one, the PMOS transistor $\mathrm{M}_{15}$ always stays "on" to prevent DC current from being leaked out to the retina tissue. By turning the $S_{4}$ to a $\operatorname{logic} 1$, the $V_{\mathrm{CA}}$ stored on $\mathrm{C}_{2}$ is applied to the gate of the NMOS transistor $\mathrm{M}_{14}$, thereby generating a cathodic current $I_{C}$ :

$$
I_{\mathrm{C}}=\frac{1}{2} \mu_{\mathrm{N}} C_{\mathrm{OX}}\left(\frac{W}{L}\right)_{14}\left(V_{\mathrm{CA}}-V_{\mathrm{TH}, \mathrm{N}}\right)^{2} \text { and thus } \frac{I_{\mathrm{C}}}{I_{\mathrm{PD}}}=\frac{\left(\frac{W}{L}\right)_{14}}{\left(\frac{W}{L}\right)_{9}}
$$

Here, the channel-length modulation effect can be reduced by enlarging the length of $\mathrm{M}_{14}$ while still making the aspect ratio $(\mathrm{W} / \mathrm{L})_{14}$ equal to $(\mathrm{W} / \mathrm{L})_{9}$. In order to generate an anodic current pulse, the conventional designs exploit an additional current-mirror branch $[4,6,12,13]$. This results in unnecessary power consumption and a large mismatch between the cathodic and anodic currents.

Under the assumption that the additional current-mirror branch to copy $I_{\mathrm{PD}}$ for $I_{\mathrm{A}}$ exits in Figure 2a, the average power that the SPStim dissipates can be approximated to:

$$
P_{\mathrm{av}} \approx \underbrace{\frac{1}{T_{\mathrm{S}}} \int_{0}^{T_{\mathrm{S}}} I_{\mathrm{PD}} \times\left(V_{\mathrm{DD}}-V_{\mathrm{SS}}\right) \mathrm{dt}}_{\text {Current Amplifier }}+\underbrace{\frac{1}{T_{\mathrm{S}}} \int_{0}^{T_{\mathrm{S}}}\left[I_{\mathrm{A}} \times V_{\mathrm{DD}}-I_{\mathrm{C}} \times V_{\mathrm{SS}}+I_{\mathrm{C}} \times\left(V_{\mathrm{DD}}-V_{\mathrm{SS}}\right)\right] \mathrm{dt}}_{\text {Pulse Shaper }}
$$

Here, $I_{\mathrm{DARK}}$ is ignored because of $I_{\mathrm{PD}}>>I_{\mathrm{DARK}}$ and $T_{\mathrm{S}}$ denotes the period of the biphasic waveform. In the second term of Equation (5), $I_{\mathrm{C}} \times\left(V_{\mathrm{DD}}-V_{\mathrm{SS}}\right)$ arises from the additional branch. Thus, the average power dissipated by all mirroring branches can be written as:

$$
P_{\text {tot,branch }} \approx N \times \frac{1}{T_{\mathrm{S}}} \int_{0}^{T_{\mathrm{S}}} I_{\mathrm{C}} \times\left(V_{\mathrm{DD}}-V_{\mathrm{SS}}\right) \mathrm{dt}
$$

where $N$ means the number of pixels simultaneously stimulated. $N$ also becomes higher in a subretinal device with high-density pixels.

By adopting the capacitor $\mathrm{C}_{1}$ to directly record the value of $V_{\mathrm{PD}}$, in this work, we remove the need for the additional branch, thus reducing both mismatch and power dissipation. As a result, the average power of the SPStim can be approximately expressed as:

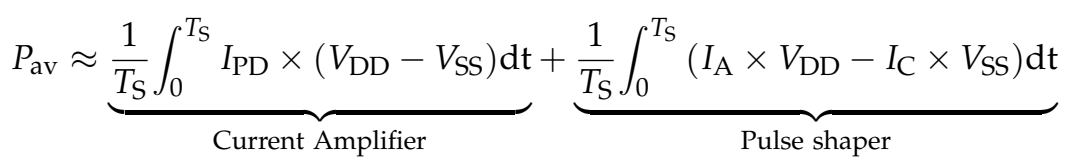

This equation shows that the proposed SPStim consumes relatively less power compared to a stimulator using the addition current-mirror branch. 
After the cathodic pulse, a logic 1 is applied to the PMOS transistor $\mathrm{M}_{5}$, and then the $V_{\mathrm{PD}}$ produces the anodic current $I_{\mathrm{A}}$ from $\mathrm{M}_{7}$. The dummy transistors $\mathrm{M}_{4}$ and $\mathrm{M}_{11}$ are also harnessed to avoid charge injection caused by the $\mathrm{M}_{5}$ and $\mathrm{M}_{12}$ switching. Consequently, the two switched-capacitor current mirrors adopted in the proposed SPStim make it possible to reduce power consumption in high-density pixel array; reduce the mismatch, which allows low residual charge on the tissue after stimulation; and widen the output dynamic range, which enables low-voltage operation.

\section{Measurement}

Figure 3 shows a micrograph of the 64-pixel stimulator array adopting the proposed architecture, and the global digital controller to produce digital signals for $S_{1}, S_{2}, S_{3}, S_{4}$ and $S_{5}$ in Figure 2a; both were fabricated in a single chip using a standard 0.35- $\mu \mathrm{m} 4 \mathrm{M} 2 \mathrm{P}$ CMOS process. This full chip occupies an active area of $4.3 \times 3.2 \mathrm{~mm}^{2}$. We first conducted a benchtop experiment to measure the transient bip-hasic current waveforms, which vary with the incident light intensity, using a light source (Model: Newport 66088-LED). Figure 4a illustrates the biphasic current waveform and its digital control signals observed with an oscilloscope (Model: Tektronix MSO4104). By changing the light intensity and supplying various digital pulses to the SPStim, we successfully generated diverse biphasic current shapes in amplitude, width, interphase and period. In this benchtop experiment, we used a 10-k $\Omega$ resistor to simply mimic the electrode resistance and set the time width for $S_{2}$ to $2 \mathrm{~ms}$ for fair comparison of the light-dependent biphasic current amplitudes.

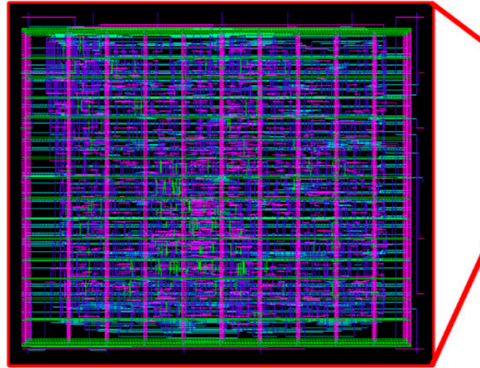

(a)

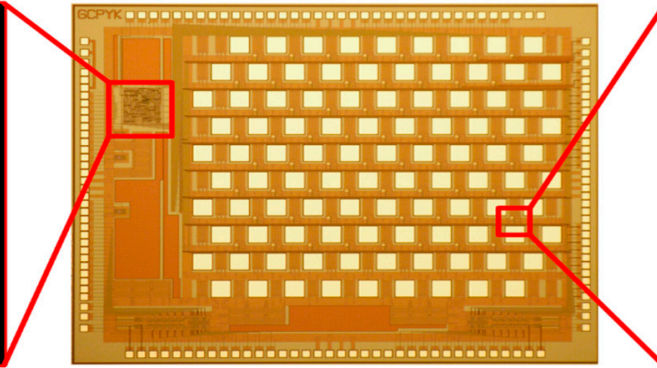

(b)

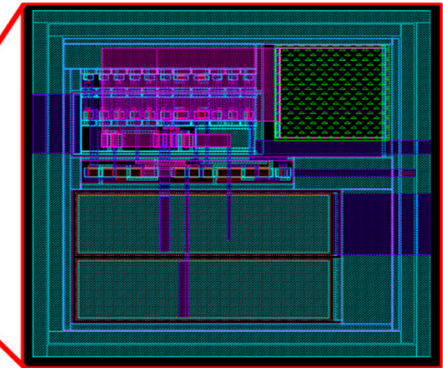

(c)

Figure 3. (a) Layout of the global digital controller embedded onto the signal chip with a 64-pixel stimulator array (its active area is gauged as $441.5 \times 425.5 \mu \mathrm{m}^{2}$ ); (b) micrograph of the 64-pixel stimulator array; (c) layout of the proposed single-pixel stimulator, which occupies an active area of $114 \times 117 \mu \mathrm{m}^{2}$.

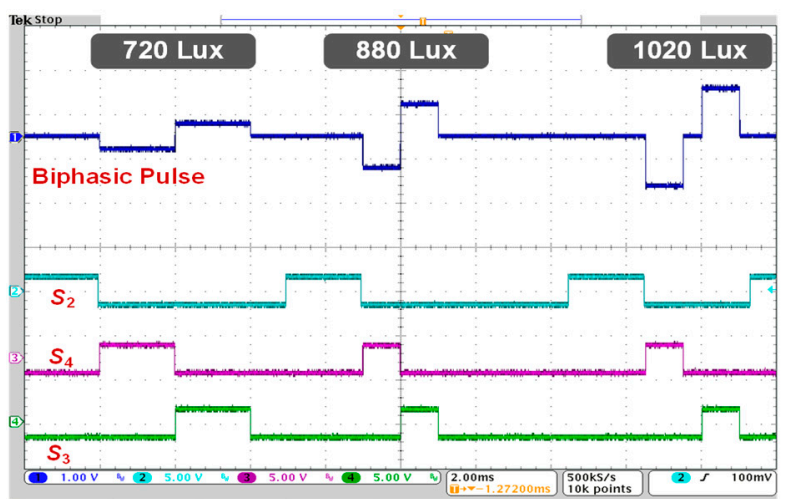

(a)

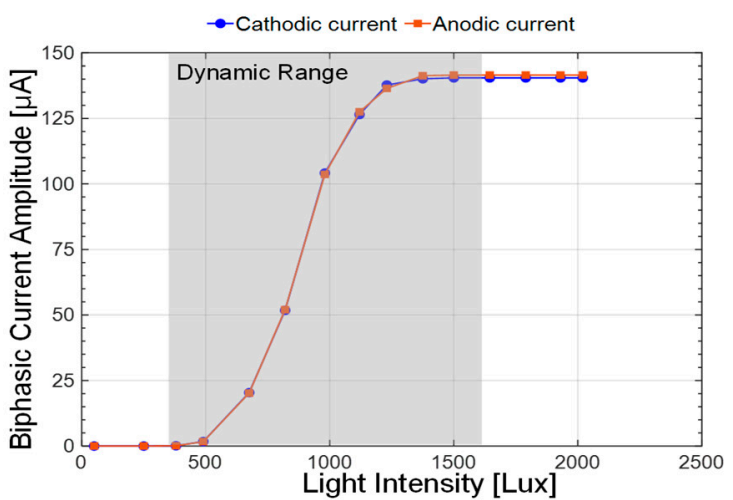

(b)

Figure 4. (a) Measured transient waveforms for the biphasic current and digital input pulses, where a $10-\mathrm{k} \Omega$ resistor was used to model the electrode resistance; (b) anodic and cathodic current amplitudes varying with incident light intensity. 
Figure $4 \mathrm{~b}$ shows the measured anodic (red line) and cathodic (blue line) amplitudes relying on the incident light intensity.

The observed results show that the biphasic stimulus current of the proposed SPStim varies from 0 to $143 \mu \mathrm{A}$ in the dynamic range from 400 to 1600 lux, where an average mismatch of $1.12 \%$ was calculated using the following equation.

$$
\text { Mismatch }=\frac{1}{N} \sum_{n=1}^{N} \frac{\left|A_{\text {anodic }}(n)-A_{\text {cathodic }}(n)\right|}{\max \left[A_{\text {anodic }}(n), A_{\text {cathodic }}(n)\right]}
$$

Here, $N, A_{\text {anodic }}$ and $A_{\text {cathodic }}$ indicate the number of measured samples, the anodic and cathodic pulse amplitudes in the dynamic range. This low mismatch is due to the switched-capacitor current mirror technique described in Figure $2 \mathrm{a}$. The charge remaining on the tissue after stimulation was completely removed by turning on the charge-balancing switch $\mathrm{M}_{15}$.

Next, we ex-vivo demonstrated the proposed SPStim using a dissected pig eyeball as shown in Figure 5. Here, it is observed that the biphasic pulse amplitudes are reduced by approximately $29 \%$ compared to the waveforms measured in Figure $4 \mathrm{a}$. This is because the incident light intensity is attenuated when passing through the pig eyeball lens [17]. In terms of Equation (1), the attenuated light reduces $\mathrm{I}_{\mathrm{DARK}}$ in the photodiode, thereby reducing the stimulation current $\mathrm{I}_{\mathrm{PD}}$. To compensate for the reduced $\mathrm{I}_{\mathrm{PD}}$, we elongated the $\mathrm{S}_{2}(\Delta \mathrm{T}$ in Equation (1)) from $2 \mathrm{~ms}$ (see Figure $4 \mathrm{a}$ ) to $2.5 \mathrm{~ms}$, and as a result, the SP-Stim could generate the same biphasic pulse amplitudes as observed in Figure $4 \mathrm{~b}$ in the same dynamic range. This demonstration shows that the customized global digital controller is necessary to adjust biphasic stimulus pulses after implantation. Finally, the overall performance of the proposed SPStim is summarized in Table 1.

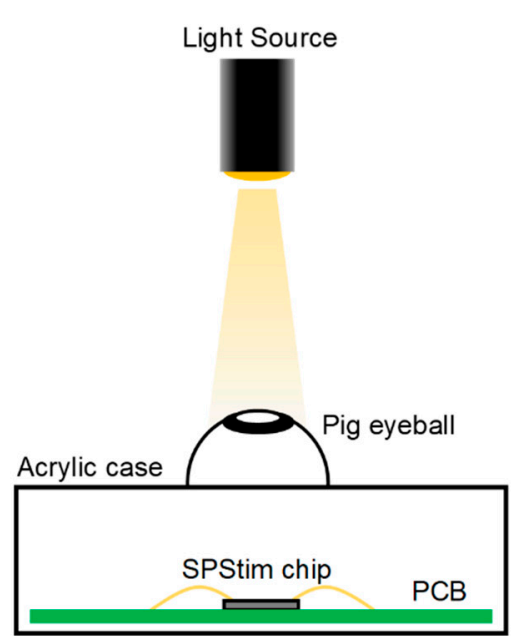

(a)

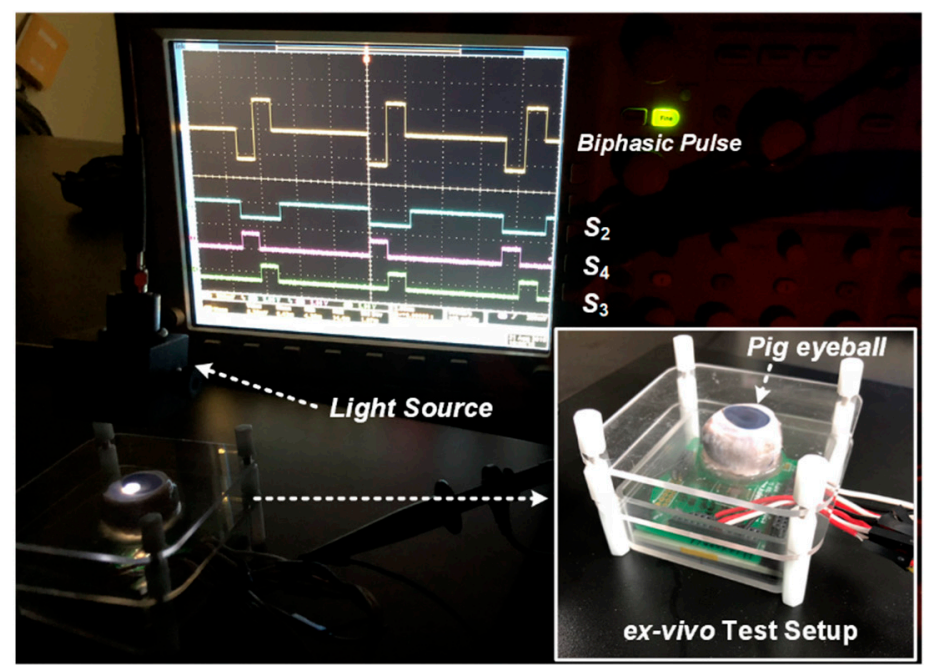

(b)

Figure 5. (a) Illustration of the ex-vivo demonstration setup; (b) ex-vivo experiment using a dissected pig eyeball. 
Table 1. Electrical performance Summary and comparison of the proposed single-pixel stimulator (SPStim).

\begin{tabular}{|c|c|c|c|c|c|c|}
\hline & [18] & [6] & [12] & [13] & {$[3,18-20]$} & This Work \\
\hline CMOS Process & $0.35-\mu \mathrm{m}$ & $0.18-\mu \mathrm{m}$ & $0.18-\mu \mathrm{m}$ & $0.35-\mu \mathrm{m}$ & $0.35-\mu \mathrm{m}$ & $0.35-\mu \mathrm{m}$ \\
\hline CNIUS ITOCESS & standard & CIS ** & CIS ** & $\mathrm{BCD}^{* * *}$ & CIS ** & standard \\
\hline Supply voltage [V] & 5 & \pm 1.65 & $0.5,1.8$ & 5,12 & \pm 2 & \pm 1.65 \\
\hline Chip area $\left[\mathrm{mm}^{2}\right]$ & $1.0 \times 2.7$ & $2.2 \times 2.2$ & $1.9 \times 1.9$ & $2.5 \times 1.2$ & $3 \times 3.5$ & $4.3 \times 3.2$ \\
\hline Single pixel area $\left[\mu \mathrm{m}^{2}\right]$ & $200 \times 200$ & $127 \times 167$ & $30 \times 30$ & $55 \times 50$ & $30 \times 30$ & $114 \times 117$ \\
\hline Dynamic range [Lux] & - & $400-1200$ & $1-1000$ & $100-250$ & $0.1-10,000$ & $400-1600$ \\
\hline Pulse amplitude $*[\mu \mathrm{A}]$ & 50-1050 & $0-343$ & $0-50$ & $0-300$ & $0-100$ & $0-143$ \\
\hline On-chip digital controller & Yes & No & Yes & No & Yes & Yes \\
\hline Pulse width * [ms] & - & - & - & - & 4 & $0.5-5$ \\
\hline Pulse period ${ }^{*}[\mathrm{~ms}]$ & - & - & 60 & - & $3-100$ & $1-50$ \\
\hline Interphase delay ${ }^{*}[\mathrm{~ms}]$ & - & - & - & - & $0 \mathrm{~ms}$ & $0-2 \mathrm{~ms}$ \\
\hline Mismatch [\%] & - & 4.85 & - & - & - & 1.12 \\
\hline Stimulation method & \multicolumn{6}{|c|}{ Charge-balanced biphasic current stimulation } \\
\hline
\end{tabular}

* Pulse: Biphasic Pulse, ${ }^{* *}$ CIS: CMOS Image Sensor, ${ }^{* * *}$ BCD: Bipolar CMOS and Double diffused MOS(DMOS).

\section{Conclusions}

A fully integrated low-power and low-mismatch SPStim was designed for subretinal implants. By adopting two switched-capacitor current mirrors for anodic and cathodic pulse generation, in this work, we achieved relatively less power consumption, compared to a SPStim using the additional current-mirror branch to generate an anodic or a cathodic stimulation pulses, and low mismatch to decrease the residual charges that can have deleterious effects on the retina. Using the customized $\mathrm{P}+/ \mathrm{N}$-well photodiode in a standard CMOS process also enabled relatively lower fabrication cost than a complex CMOS images sensor process. The 64-pixel stimulators employing the proposed architecture were fabricated in a standard $0.35-\mu \mathrm{m}$ CMOS process and ex-vivo tested with a dissected pig eyeball. From the measured results, the device achieves a maximum biphasic pulse amplitude of $143 \mu \mathrm{A}$, an output dynamic range of $86.7 \%$, and a mismatch of $1.12 \%$ on average between the anodic and cathodic pulses. The customized global digital controller, for producing diverse biphasic stimulus currents in amplitude, width, interphase delay, and period, was embedded on the same chip with the 64-pixel stimulators and used to compensate for the attenuated light intensity passing through the pig eyeball lens in ex-vivo experiments. In future work, a refined 64-pixel stimulator array will functionalize the wireless power and data telemetry that we are developing now.

Author Contributions: The individual contributions of authors are follows: H.K. designed the stimulator chip and wrote the manuscript. W.H.A. designed the on-chip digital controller and took part in the manuscript revision. S.-W.K. conducted the ex-vivo experiment using the dissected pig eyeball. J.K. managed the project.

Funding: This research was partially supported by the National Research Foundation of Korea (Grant No. NRF-2017M3A9E2056461) and the Gachon University Gil Medical Center (Grant No. 2016-16).

Acknowledgments: The authors are grateful to the IC Design Education Center (IDEC) for chip fabrication.

Conflicts of Interest: The authors declare no conflict of interest.

\section{References}

1. Ortmanns, M.; Rocke, A.; Gehrke, M.; Tiedtke, H. A 232-Channel Epiretinal Stimulator ASIC. IEEE J. Solid-State Circuits 2007, 42, 2946-2959. [CrossRef]

2. Chen, K.; Yang, Z.; Hoang, L.; Weiland, J.; Humayun, M.; Liu, W. An Integrated 256-Channel Epiretinal Prosthesis. IEEE J. Solid-State Circuits 2010, 45, 1946-1956. [CrossRef]

3. Rothermel, A.; Liu, L.; Aryan, N.P.; Fisher, M.; Wuenschmann, J.; Kibbel, S.; Harscher, A. A CMOS Chip with Active Pixel Array and Specific Test Features for Subretinal Implantation. IEEE J. Solid-State Circuits 2009, 44, 290-300. [CrossRef] 
4. Ng, D.C.; Furumiya, T.; Yasuoka, K.; Uehara, A.; Kagawa, K.; Tokuda, T.; Nunoshita, M.; Ohta, J. Pulse frequency modulation based CMOS image sensor for subretinal stimulation. IEEE Trans. Circuits Syst. II Express Briefs 2006, 53, 487-491. [CrossRef]

5. Boinagrov, D.; Lei, X.; Goetz, G.; Kamins, I.T.; Mathieson, K.; Galambos, L.; Harris, S.J., Jr.; Palanker, D. Photovoltaic Pixels for Neural Stimulation: Circuit Models and Performance. IEEE Trans. Biomed. Circuits Syst. 2016, 10, 85-97. [CrossRef] [PubMed]

6. Park, H.; Shim, S.; Jeong, J.; Kim, S. A Multi-photodiode Array-based Retinal Implant IC with On/off Stimulation Strategy to Improve Spatial Resolution. JSTS J. Semicond. Technol. Sci. 2017, 17, 35-41. [CrossRef]

7. Ayton, L.N.; Blamey, P.J.; Guymer, R.H.; Luu, C.D.; Nayagam, D.A. First-in-Human Trial of a Novel Suprachoroidal Retinal Prosthesis. PLoS ONE 2014, 9, e115239. [CrossRef] [PubMed]

8. Zrenner, E.; Bartz-Schmidt, K.U.; Besch, D.; Gekeler, F.; Koitschev, A.; Sachs, H.G.; Stingl, K. The Subretinal Implant ALPHA: Implantation and Functional Results, Artificial Vision: Clinical Guide; Springer international Publishing: Berlin, Germany, 2017; pp. 65-83.

9. Cheng, D.L.; Greenburg, P.B.; Borton, D.A. Advances in Retinal Prosthetic Research: A Systematic Review of Engineering and Clinical Characteristics of Current Prosthetic Initiatives. Curr. Eye Res. 2017, 42, 334-347. [CrossRef] [PubMed]

10. Merrill, D.R.; Bikson, M.; Jefferys, J.G.R. Electrical stimulation of excitable tissue: Design of efficacious and safe protocols. J. Neurosci. Method 2004, 141, 171-198. [CrossRef] [PubMed]

11. Humayun, M.S.; Juan, E., Jr.; Weiland, J.D.; Dagnelie, G.; Katona, S.; Greenberg, R.; Suzuki, S. Pattern electrical stimulation of the human retina. Curr. Eye Res. 1999, 39, 2569-2576. [CrossRef]

12. Chih-Lin, L.; Chih-Cheng, H. A 0.5 V/1.8 V High Dynamic Range CMOS Imager for Artificial Retina Applications. IEEE Sens. J. 2015, 15, 6833-6838.

13. Oh, S.; Ahn, J.; Lee, S.; Ko, H.; Seo, J.; Goo, Y.; Cho, D. Light-Controlled Biphasic Current Stimulator IC Using CMOS Image Sensors for High-Resolution Retinal Prosthesis and in-vitro Experimental Results with rd1 Mouse. IEEE Trans. Biomed. Eng. 2015, 62, 70-79. [CrossRef]

14. Kartikeya, M.; Ralph, E.; Nitish, T.; Gert, C. Which photodiode to Use: A Comparison of CMOS-Compatible Structure. IEEE Sens. J. 2009, 9, 752-760.

15. Köklü, G.; Etienne-Cummings, R.; Leblebici, Y.; Micheli, G.; Carrara, S. Characterization of standard CMOS compatible photodiodes and pixels for lab-on-chip devices. In Proceedings of the IEEE International Symposium on Circuits and Systems, Beijing, China, 19-23 May 2013; pp. 1075-1078.

16. Zhang, L.; Wang, Z.-H.; Li, Y.-M.; Zhang, C.; Wang, Z.-H.; Chen, H.-H. Clock generator and OOK modulator for RFID application. J. Zhejiang Univ. Sci. 2005, 6A, 1051-1053.

17. Seo, H.; Nam, D.; Lee, J.; Park, S.; Kim, Y.; Kim, S.; Chung, T.; Inoue, M.; Kim, T. Macular photostress and visual experience between microscope and intracameral illumination during cataract surgery. J. Cataract Refract. Surg. 2018, 44, 190-197. [CrossRef] [PubMed]

18. Tokuda, T.; Hiyama, K.; Sawamura, S.; Sasagawa, K.; Terasawa, T.; Nishida, K.; Kitaguchi, Y.; Fujikado, T.; Tano, Y.; Ohta, J. CMOS-Based Multichip Networked Flexible Retinal Stimulator Designed for Image-Based Retinal Prosthesis. IEEE Trans. Electron Devices. 2009, 56, 2577-2585. [CrossRef]

19. Rothermel, A. Recent results with subretinal stimulation. In Proceedings of the IEEE Biomedical Circuits and Systems Conference (BioCAS), Lausanne, Switzerland, 22-24 October 2014; pp. 220-223.

20. Liu, L.; Wiinschmann, J.; Aryan, P.N.; Zohny, A.; Fischer, M. An ambient light adaptive subretinal stimulator. In Proceedings of the 2009 ESSCIRC, Athens, Greece, 14-18 September 2009; pp. 420-423.

(C) 2019 by the authors. Licensee MDPI, Basel, Switzerland. This article is an open access article distributed under the terms and conditions of the Creative Commons Attribution (CC BY) license (http://creativecommons.org/licenses/by/4.0/). 